\title{
La investigación social en la esfera de la discapacidad: realidad y tendencias
}

Social research in the field of disability: reality and trends

\section{Palabras clave:}

Discapacidad, investigación social, planificación, políticas públicas, gestión del conocimiento.

\section{Keywords}

Disability, social research, planning, public policies, knowledge management.

\section{Eduardo Díaz Velázquez <ediaz@siis.net>}

Centro Español de Documentación sobre Discapacidad (CEDD). España

\section{Introducción ${ }^{1}$}

Este texto tiene su origen en una ponencia que me invitó a impartir el CERMl a finales del año 2014 en unas jornadas sobre investigación social y discapacidad, cuya temática no ha perdido vigencia sino que por el contrario ha ganado con el paso del tiempo entidad y actualidad, de tal modo que he considerado pertinente plasmarla en una publicación escrita y, particularmente, en la sección Tribuna de la Revista Española de Discapacidad (REDIS), la cual es un valioso espacio de reflexión, intercambio y generación de conocimiento científico en materia de discapacidad.

$Y$ es que, cuando nos acercamos a la discapacidad desde un punto de vista científico o técnico, es muy relevante pensar y reflexionar acerca de cómo establecemos ese acercamiento, de cómo enfocamos la discapacidad desde una (supuesta) distancia científica y, en particular, desde la perspectiva de la investigación social.

1. Este texto, debidamente actualizado, está basado en la ponencia "La investigación social en la esfera de la discapacidad, realidad y tendencias" presentada en las Jornadas sobre Investigación social y Diversidades Humanas: Generando conocimiento sobre la discapacidad, organizadas por el Comité Español de Representantes de Personas con Discapacidad (CERMI) e ILUNION Consultoría y celebradas el 12 de diciembre de 2014 en Madrid.

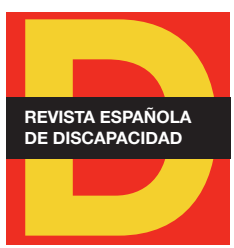

Para citar:

Díaz Velázquez, E. (2018): “La investigación social en la esfera de la discapacidad: realidad y tendencias". Revista Española de Discapacidad, 6 (II): 191-203.

Doi: <https://doi.org/10.5569/23405104.06.02.10> 
Haciendo una mirada retrospectiva, en los últimos años podemos constatar que los investigadores sociales (tanto del ámbito académico como del profesional), particularmente en España, cada vez se interesan más en estudiar la discapacidad: crece el número de artículos en revistas científicas especializadas, su presencia es cada vez mayor en diferentes foros de ciencias sociales, en la universidad es creciente el número de tesis doctorales, trabajos de fin de máster o trabajos de fin de grado sobre esta temática... Al mismo tiempo, cada vez hay mayor demanda en conocer de forma más o menos rigurosa cuál es la realidad social de las personas con discapacidad en diferentes ámbitos: el empleo, la educación, la participación social y política, la accesibilidad, el acceso a la salud, etc.

En suma, la discapacidad cada vez es un campo más relevante de estudio por parte de la sociología y de otras ciencias sociales. Y eso se debe, en parte, a la consolidación de una visión de la discapacidad como cuestión social y como producto social (Díaz Velázquez, 2010). También ha influido el papel que han tenido las entidades del sector, con el CERMI y Fundación ONCE a la cabeza, en visibilizar esa problemática social (la desigualdad y exclusión por motivo de discapacidad) y en proporcionar herramientas para analizarla: ya sea mediante el desarrollo de estudios sociales o a través del trabajo continuo con el Instituto Nacional de Estadística y otras instancias públicas para que la discapacidad esté presente de manera transversal en las fuentes estadísticas, así como en las diferentes políticas públicas.

$\mathrm{Y}$, efectivamente, hemos avanzado mucho. Hace quince años (cuando yo me iniciaba profesionalmente en este campo) era impensable poder disponer de tantas fuentes estadísticas que contuvieran datos de discapacidad como tenemos ahora. Y, lo que es más importante, no estadísticas específicas sobre discapacidad, sino estadísticas generales que, en mayor o menor medida y de forma más o menos imperfecta, desagregan sus datos por la variable discapacidad, como la serie El Empleo de las Personas con Discapacidad (EPD)2; el Salario de las Personas con Discapacidad (SPD) ${ }^{3}$, la Encuesta de Integración y Salud (EISS) o la más novedosa (y en otro ámbito) Estadística de los declarantes con discapacidad del Impuesto sobre la Renta de las Personas Físicas (de la Agencia Tributaria) ${ }^{4}$, si bien queda aún mucho camino por recorrer. De la misma forma, a nivel europeo la Oficina Europea de Estadística, Eurostat, recopila un importante número de datos desagregados por la variable discapacidad en diferentes temáticas y de forma comparada entre países de la UE-28, procedentes de diferentes fuentes estadísticas europeas 5 .

También está previsto que en el año 2020 tengamos disponibles los datos de la nueva Encuesta de Discapacidad, Autonomía Personal y situaciones de Dependencia (EDAD) del Instituto Nacional de Estadística, que actualmente se encuentra en fase de diseño y preparación.

Todas estas operaciones estadísticas son buena prueba de los avances en la visibilización de la realidad social de las personas con discapacidad y de la necesidad de conocer su situación de forma más precisa para poder intervenir mejor sobre ella. Sin embargo, aún queda una tarea pendiente, un objetivo marcado con respecto a las fuentes estadísticas, buscando su máxima optimización: el de la transversalidad; esto es, que se pudiera conocer información desagregada del colectivo de personas con discapacidad para todas y cada una de las fuentes estadísticas existentes. De buscar soluciones operativas para dar respuesta a

2. Véase: http://www.ine.es/dyngs/INEbase/es/operacion.htm?c=Estadistica_C\&cid=1254736055502\&menu=ultiDatos\&idp=1254735573175. 3. Véase: http://www.ine.es/dyngs/INEbase/es/operacion.htm?c=Estadistica_C\&cid=1254736176911\&menu=ultiDatos\&idp=1254735573175.

4. Véase: https://www.agenciatributaria.es/AEAT.internet/datosabiertos/catalogo/hacienda/Estadistica_sobre_discapacidad_declaraciones_IRPF. shtml.

5. Véase: https://ec.europa.eu/eurostat/web/health/disability/data/database. 
este objetivo se han ocupado diferentes iniciativas, entre las que cabe destacar, a nivel nacional, el Grupo de Trabajo sobre estadísticas y discapacidad constituido en el CERMI en el año 2013, en el que he tenido el honor de participar. A nivel internacional, destaca el trabajo realizado por el Grupo de Washington ${ }^{6}$, que ha diseñado una batería de preguntas (en versión amplia o reducida) para identificar a la población con discapacidad de manera transversal en encuestas generalistas y poder así obtener datos desagregados por discapacidad y tipos de discapacidad (entendida como limitaciones en la actividad).

Si bien las estadísticas son fundamentales para conocer la realidad, no considero que toda la investigación social en la esfera de la discapacidad tenga que quedar reducida a ellas. Es por esa razón que quisiera contextualizar la realidad actual de los estudios sociales sobre discapacidad en España y plantear mi punto de vista de cuáles deberían ser los caminos a seguir. Para ello, creo que debemos realizarnos algunas preguntas básicas de partida:

- ¿Por qué investigar sobre discapacidad?

- ¿Para qué investigar?

- ¿Qué investigar?

- ¿Cómo investigar?

- ¿Con qué recursos?

\section{2. ¿Por qué investigar?}

Las convicciones éticas y sociales acerca de por qué investigar la realidad de las personas con discapacidad se han materializado en los últimos años en diferentes preceptos normativos. Considero, y supongo que muchos coincidiremos en ello, que el marco teórico, analítico y conceptual del que debemos partir se encuentra en la Convención sobre los derechos de las personas con discapacidad, en el modelo social así como en la perspectiva de derechos y de ciudadanía, que se puede traducir en la investigación social en analizar si el acceso, no sólo formal sino principalmente real y efectivo, de las personas con discapacidad a los derechos de ciudadanía se lleva a cabo y se hace en condiciones de igualdad con el resto de la población (Díaz Velázquez, 2017).

La investigación social sobre discapacidad y, en particular, este enfoque, está fundamentado en la propia Convención, en concreto en su artículo 317, que insta a los Estados Partes a recopilar datos estadísticos y realizar investigación sobre discapacidad que les permita formular y aplicar sus políticas sobre discapacidad.

6. Véase: http://www.washingtongroup-disability.com/.

7. Artículo 31: Recopilación de datos y estadísticas

1. Los Estados Partes recopilarán información adecuada, incluidos datos estadísticos y de investigación, que les permita formular y aplicar políticas, a fin de dar efecto a la presente Convención. En el proceso de recopilación y mantenimiento de esta información se deberá:

a) Respetar las garantías legales establecidas, incluida la legislación sobre protección de datos, a fin de asegurar la confidencialidad y el respeto de la privacidad de las personas con discapacidad;

b) Cumplir las normas aceptadas internacionalmente para proteger los derechos humanos y las libertades fundamentales, así como los principios éticos en la recopilación y el uso de estadísticas. 
La traslación normativa de la Convención a nuestro país es un hecho, aunque no podamos aún afirmar que esta se haya llevado a cabo de forma plena, existiendo aún preceptos pendientes de traslación como, fundamentalmente, el artículo 12 en lo relativo al ejercicio de la capacidad jurídica (Real Patronato sobre Discapacidad, 2012). De la misma forma, su espíritu también ha sido trasladado a nuestras políticas públicas. Así, el Plan de Acción de la Estrategia Española de Discapacidad 2014-2020 tenía entre sus objetivos estratégicos "garantizar la plena igualdad de las personas con discapacidad en el ejercicio de todos sus derechos con el resto de los ciudadanos" (DGPAD, 2014), y entre sus actuaciones contemplaba la realización de estudios específicos, así como el mayor desarrollo de las fuentes estadísticas disponibles. Además, otro de sus objetivos operativos se centra en promover un sistema de información accesible sobre discapacidad (desarrollado entre las actuaciones 81 y 89), que además de promover la investigación y la producción del conocimiento sobre discapacidad, se encargara de su gestión y divulgación. En algunas de estas tareas hemos participado activamente desde el Centro Español de Documentación sobre Discapacidad (CEDD), como en el desarrollo de esta revista o en la creación de Riberdis, de los que nos ocuparemos más adelante.

\section{3. ¿Para qué investigar?}

La segunda pregunta que nos tenemos que hacer es: ¿para qué investigar sobre discapacidad? Creo que la finalidad de la investigación social tiene que ser eminentemente práctica ${ }^{8}$ y ha de ser estar orientada a la transformación social: conocer para transformar. Se ha de analizar la realidad para planificar las políticas públicas e intervenciones necesarias que mejoren la situación de las personas con discapacidad.

En mi experiencia en la consultoría social, en algunas ocasiones tenías la impresión de que algunos estudios se guardaban en un cajón, o bien se publicaban y ahí acababa su recorrido, pero que no influían ni transformaban la práctica, pues no eran utilizados para la planificación de las políticas e intervenciones. Seguramente no siempre nosotros como investigadores sabemos mostrar todo el potencial y utilidad práctica de estos trabajos, pero también a veces las vías de conexión entre la investigación y la planificación no han sido las mejores, pues esta no siempre se ha basado suficientemente en la evidencia empírica.

Las investigaciones sociales sobre discapacidad han de servir para planificar las políticas públicas, para priorizar algunos temas sobre otros, para poder gestionar mejor los recursos y poder ponderar mejor las partidas de gasto que se destinen a unos recursos o servicios frente a otros. Han de ser útiles para la toma de decisiones (la investigación social no sirve si no se difunde, si no se utiliza y se guarda en un cajón). Pero para ello también la investigación social que se realice sobre discapacidad ha de ser rigurosa, seria, exhaustiva y propositiva. Y tenemos que hacer visible su propia utilidad.

2. La información recopilada de conformidad con el presente artículo se desglosará, en su caso, y se utilizará como ayuda para evaluar el cumplimiento por los Estados Partes de sus obligaciones conforme a la presente Convención, así como para identificar y eliminar las barreras con que se enfrentan las personas con discapacidad en el ejercicio de sus derechos.

3. Los Estados Partes asumirán la responsabilidad de difundir estas estadísticas y asegurar que sean accesibles para las personas con discapacidad y otras personas.

8. Aunque no únicamente, ya que la producción teórica sobre discapacidad desde la sociología y otras ciencias sociales ayuda a repensar el fenómeno y a analizar de forma crítica cómo se construye este socialmente, lo que también repercute en la visión social de la discapacidad y en las propias políticas que se desarrollen. 


\section{4. ¿Qué investigar?}

Esta pregunta es susceptible de una amplia variedad de respuestas. Los expertos en esta materia podríamos identificar algunos temas prioritarios para una hipotética agenda de investigación sobre discapacidad y a buen seguro no coincidir en algunos (o muchos) de ellos. Hay múltiples temáticas a abordar en la investigación social sobre discapacidad. Pero, ¿cuáles podemos considerar más prioritarias? No quisiera yo definir una serie de temas que investigar, pero sí que me gustaría señalar que es necesario identificar y priorizar los principales fenómenos sobre los que investigar: algo que podríamos llamar Agenda de Investigación (Social) sobre Discapacidad, la cual, siguiendo los postulados de la Convención, identificara las temáticas más relevantes que tratar y las priorizara, en base a criterios objetivos y al saber experto.

En octubre de 2014, apenas dos meses antes de realizar la ponencia que da origen a este texto, me invitaron a participar en un encuentro sobre investigación social y discapacidad en Costa Rica, organizado por el Consejo Nacional de Rehabilitación y Educación Especial (CNREE), que tenía como objetivo "potenciar la generación de conocimiento en el campo de la discapacidad por parte de los principales centros e institutos de investigación del país, que sustente la toma de decisiones y que se traduzca en soluciones innovadoras capaces de incidir en la transformación social, para la inclusión efectiva de la población con discapacidad en el desarrollo del país". Probablemente, la investigación social sobre discapacidad en nuestro país tenga una trayectoria algo mayor, pero me parece muy interesante el planteamiento de ese encuentro, que era perfilar a nivel epistemológico, conceptual y metodológico, hacia dónde debía dirigirse la investigación sobre discapacidad en Costa Rica, desde una perspectiva de derechos humanos, con un doble objetivo (al que se acercaron mediante el trabajo conjunto de investigadores, planeadores, técnicos y expertos): formular una agenda nacional de investigación sobre discapacidad y tratar de incorporar transversalmente la discapacidad en los estudios sociales que allí se efectúan. Y todo ello, para sustentar la toma de decisiones en la acción pública.

De la misma manera, creo que es necesario elaborar algo así como una Agenda Nacional de Investigación Social sobre Discapacidad, formando parte en su planificación diferentes agentes sociales del ámbito público y de la sociedad civil, a saber: las propias personas con discapacidad y sus entidades representativas, investigadores de las universidades, técnicos de la administración pública, expertos de reconocido prestigio, de tal modo que se pudieran conformar grupos de trabajo que priorizaran temáticas sobre las que investigar (e intervenir) y trabajar sobre ellas. Incorporar la discapacidad y la accesibilidad explícitamente en las sucesivas convocatorias del Programa estatal I+D+i, hoy en día ausentes, es fundamental.

En definitiva, es necesario establecer cuáles serían las líneas de investigación prioritarias y cuáles las estrategias de investigación para llevarlas a cabo (y aquí no me refiero sólo a nivel metodológico y de producción de conocimiento, sino también a su gestión, difusión y divulgación).

Por apuntar, humildemente, algunas líneas de investigación relevantes (no tanto temáticas), creo que sería prioritario:

- el género (tanto estudios de género -por ejemplo, sobre violencia de género y discapacidad, de lo que se conoce poco- como su enfoque transversal a los diferentes estudios); 
- el empleo y la (in)actividad, analizando tanto el acceso como las condiciones laborales, así como los factores que facilitan u obstaculizan el acceso al empleo;

- la situación de la infancia con discapacidad y sus familias, determinando el impacto (no sólo en términos económicos) de la discapacidad en los menores y sus familias;

- las desigualdades socioeconómicas (en términos de ingresos y gastos), considerando la discapacidad como un factor de estratificación social;

- la movilidad social de las personas con discapacidad (un tema inexplorado y de gran calado, que respondería a la pregunta: ¿influye la discapacidad en la movilidad entre clases sociales? ¿de qué manera?);

- las trayectorias educativas y de qué manera el sistema educativo facilita o dificulta la inclusión del alumnado con necesidades educativas especiales;

- las necesidades sociales (incluidas las sanitarias) del colectivo y de aquellos grupos específicos;

- la accesibilidad (desde un sentido amplio);

- los contextos de multiexclusión (inmigración, minorías étnicas, medio penitenciario, etc.)

Temáticas hay muchas, pero es importante establecer (al menos) dos criterios de investigación: la comparabilidad y la continuidad temporal. Esto es, que los disability studies sean comparativos (con otros países, con la población sin discapacidad, entre regiones, por diferentes variables como el sexo o la edad, etc.), pero que también sean longitudinales, para poder analizar la evolución de los fenómenos en el tiempo.

\section{5. ¿Cómo investigar?}

Como decía, es importante desarrollar estudios longitudinales para poder contemplar la evolución de los principales fenómenos sociales relacionados con la discapacidad a lo largo del tiempo. Para poder conocer la situación, pero también para saber si las políticas que se implementan están siendo efectivas o no, para en su caso modificarlas.

También es necesario que se desarrollen estudios comparativos; por un lado, a nivel geográfico: comparativas autonómicas, internacionales (UE, OCDE); pero también comparar entre población con y sin discapacidad, para poder medir las desigualdades existentes por motivo de discapacidad e identificar mejor las barreras sociales que experimenta el colectivo en su acceso a las diferentes dimensiones de la realidad social. Metodológicamente, tenemos que tratar de combinar las estrategias metodológicas cuantitativa y cualitativa. La triangulación o el pluralismo metodológico (Cea D' Ancona, 2001: 51-59) se antoja imprescindible para abordar la realidad social de las personas con discapacidad de manera plena, para conocer pero también comprender los fenómenos. Una única estrategia metodológica nos puede dar una visión válida de la realidad, pero parcial. Por ejemplo, conocer el dato estadístico de que el $83,5 \%$ del alumnado con discapacidad en el Curso 2016/17 estaba matriculado en centros ordinarios es muy importante (Ministerio 
de Educación, 2018), pero si no analizamos la vivencia por parte del alumnado con discapacidad de esos procesos de inclusión educativa difícilmente vamos a conocer si realmente existe o no educación inclusiva o si a lo que estamos asistiendo es a una mera incorporación formal del alumnado con discapacidad en el aula ordinaria, y no a una inclusión real.

Asimismo, sería importante facilitar la participación de las propias personas con discapacidad en los procesos de investigación social, no siendo solamente ‘objeto’ de investigación sino sujetos activos de la misma.

De todo esto, creo que las tendencias prioritarias a las que apunta la investigación social sobre discapacidad serían las siguientes:

a. La creación de sistemas de indicadores y estadísticas sobre discapacidad, como los observatorios o los cuadros de mando, que recopilen, sistematicen, analicen y difundan los principales datos estadísticos sobre discapacidad. Entre algunos de ellos destacan el sistema de indicadores realizado por Huete (2013) sobre discapacidad y exclusión, que a su vez ha sido utilizado en los diferentes Informes Olivenza (Observatorio Estatal de la Discapacidad, 2017 y anteriores), así como el sistema de indicadores desarrollado por ODISMET (2014), en cuyo origen tuve la oportunidad de participar, o el sistema de indicadores para analizar el acceso a la condición de ciudadanía de las personas con discapacidad y la desigualdad por razón de discapacidad (Díaz Velázquez, 2017: 94-98; 553-561). Establecer un sistema de indicadores consensuado y que pueda ser actualizado periódicamente podría ser una de las iniciativas prioritarias que se enmarcara en esa Agenda Nacional de Investigación Social en Discapacidad.

b. El análisis y evaluación de las políticas públicas, servicios e intervenciones sobre discapacidad: en términos de cobertura, de impacto, coste-efectividad, etc., que analicen el grado de inclusión así como el ejercicio efectivo y cumplimiento real de los derechos de las personas con discapacidad que otorga nuestro ordenamiento jurídico. Una evaluación de este tipo, desde el enfoque de las 'políticas basadas en la evidencia' y con la participación de las propias personas con discapacidad, necesita una importante inversión tanto de recursos como de tiempo.

c. El estudio sobre realidades emergentes dentro del colectivo de personas con discapacidad. La discapacidad es un fenómeno variable, tanto en sus manifestaciones, como en sus efectos y consecuencias. Hay muchas realidades de la discapacidad que tal vez queden fuera de las estadísticas oficiales, discapacidades emergentes producidas por el entorno social, realidades cambiantes, nuevos fenómenos o contextos sociales y económicos de riesgo en una sociedad como la nuestra, en la que los cambios sociales se suceden con rapidez, por lo que habrá que abordarlas desde planteamientos más cualitativos, para tratar de adelantarse a ellas y, lo más importante, a sus posibles efectos adversos.

Estas tres tendencias nos indican que tan importante papel va a jugar la producción de conocimiento, como su gestión y difusión, esto es, cómo comunicar y poner a disposición de públicos muy diferentes (profesionales especializados, personas con discapacidad, público en general) la información existente. Y, al mismo tiempo, nos pone frente al reto de la transferencia de los conocimientos obtenidos en la investigación al campo de la planificación de las políticas públicas y de la acción social, tanto en el ámbito público como en el tercer sector. Esto es, cómo las investigaciones sociales que se realicen en materia de discapacidad nos van a ayudar a planificar las políticas pero también a intervenir mejor. 


\section{6. ¿Con qué recursos?}

Esta pregunta es fundamental, y a todos nos gustaría tener la mejor respuesta. La crisis económica ha supuesto en los últimos años una importante reducción del presupuesto público destinado a la investigación social en materia de discapacidad (así como a la investigación en general y a las políticas de discapacidad en conjunto), que aún no se ha recuperado. Aunque para llevar a cabo estas actuaciones es necesario contar con recursos económicos suficientes, tenemos que tener presente que ante la posibilidad de contar con recursos escasos, es fundamental saber optimizarlos y administrarlos de la mejor forma posible.

La realidad es que al tiempo que, como decíamos, cobraba un mayor protagonismo la temática de la discapacidad en la investigación social, en el contexto de crisis económica y de restricción presupuestaria se ha dispuesto de menos fondos para financiar investigaciones sociales, o incluso investigación en general, dada la reducción años atrás del peso de la l+D+i tanto en los presupuestos como en el PIB. También la práctica desaparición de las obras sociales de las cajas de ahorros tuvo un impacto negativo en la acción social del tercer sector y en la financiación de estudios sociales liderados por las entidades no lucrativas.

Desde el punto de vista de la planificación de la investigación social sobre discapacidad, es importante saber bien qué queremos investigar, priorizando los recursos económicos, y cómo queremos investigarlo, optimizando los recursos existentes, como puede ser la disposición de cada vez más fuentes estadísticas en las que se desagregue por la variable discapacidad (en lugar de encargar estudios de encuesta ad hoc, siempre que sea posible).

El dilema, sobre todo si hay recursos escasos, puede estar en: o bien financiar de manera escasa un número amplio de estudios, o bien financiar suficientemente pocos trabajos, pero que respondan a fines $u$ objetivos estratégicos de la Administración (a ser posible, decididos de manera objetiva y participada por comités de expertos independientes). En ese sentido, la segunda opción, en orden de garantizar la calidad y utilidad práctica de los estudios, se antoja como la más adecuada, siguiendo las ya mencionadas líneas de investigación prioritarias que considero que podrían ser marcadas en una agenda de investigación con una dotación presupuestaria específica.

En ese sentido, como ya indicaba, las convocatorias de ayudas a proyectos de los programas estatales de I+D+i, sobre todo aquellos orientados a dar respuesta a los retos de la sociedad, del Ministerio de Ciencia, Innovación y Universidades, podrían contemplar entre sus ejes temáticos la discapacidad y la accesibilidad. 


\section{Es necesario investigar, pero también gestionar el conocimiento y divulgarlo}

Por lo tanto, decidimos qué investigar, obtenemos los recursos, investigamos... ¿Es suficiente? Creo que no. Como apuntábamos, en la actualidad y ante la avalancha de información de nuestras sociedades, que genera 'infoxicación' (esto es, intoxicación por exceso de información), es tan importante producir conocimiento como poder gestionarlo, facilitando las herramientas para poder y saber divulgarlo, así como para crear transferencias a los contextos reales de planificación e intervención.

Para difundir, compartir y divulgar el conocimiento, se hacen imprescindibles los recolectores de datos y de información, como pueden ser los repositorios temáticos, los observatorios (fundamentales para garantizar el acceso público a los datos de una manera sencilla) y otras herramientas de divulgación que fácilmente puedan estar disponibles en la web: revistas especializadas, foros de investigación, etc. Además, las nuevas tecnologías y las redes sociales pueden ayudarnos a divulgar y acercar ese conocimiento a la población, sabiendo previamente cómo hacerlo accesible a los diferentes públicos potencialmente interesados, ya sean más o menos especializados.

Las nuevas tecnologías de la información han modificado totalmente los usos de los investigadores en lo que se refiere al acceso y el tratamiento de las fuentes documentales y estadísticas, así como las posibilidades de obtención de información sobre estudios que se están desarrollando paralelamente en otros lugares del mundo.

Si bien ahora existe una mayor inmediatez para disponer de información, la enorme capacidad de los buscadores no siempre facilita acceder a los trabajos más relevantes bajo criterios de rigor científico. Esto requiere un mayor trabajo de gestión del conocimiento que centralice aquellos documentos y estudios en acceso abierto en repositorios temáticos, que facilitarían su búsqueda al investigador. Y, en el caso de las fuentes estadísticas, de observatorios que recopilen los datos, los difundan y produzcan información cualitativa, elaborada, que permita interpretar la realidad.

Al mismo tiempo, deben mantenerse y consolidarse los espacios y foros de reflexión entre investigadores, técnicos y personas con discapacidad: seminarios, simposios, jornadas, pero también grupos focales de trabajo que generen los vínculos y transferencias entre la investigación, la planificación y la intervención. Las herramientas tecnológicas de gestión del conocimiento deben facilitar el debate, el análisis y la reflexión, para contextualizar e interpretar el fenómeno social de la discapacidad, para identificar tendencias, intercambiar conocimientos y debatir ideas.

En ese sentido, es importante que el conocimiento se produzca de manera compartida y colaborativa y que se difunda siguiendo la filosofía del Open Access, utilizando asimismo los canales de comunicación más adecuados para su divulgación entre el público interesado: investigadores, técnicos y profesionales, personas con discapacidad, público no especializado, etc. Para ello, el papel de las redes sociales en la actualidad es fundamental, aunque no se encuentran exentas de los peligros de 'infoxicación' que mencionábamos antes. 
Fieles a esta perspectiva hemos trabajado estos años en el CEDD en el campo de la discapacidad (partiendo de la base del trabajo realizado por el SIIS - Centro de Documentación y Estudios en otros campos de las políticas sociales).

En los últimos años, en el CEDD ha ido creciendo en importancia el trabajo de investigación y producción de conocimiento y asesoramiento técnico especializado a las instituciones, en particular al Real Patronato (con estudios específicos, evaluaciones de políticas públicas, análisis de datos), al mismo tiempo que hemos innovado en la gestión del conocimiento en materia de discapacidad impulsando nuevos productos que permitían poner a disposición de los investigadores y los profesionales el conocimiento existente sobre discapacidad. Entre esos productos, destacan:

- La Revista Española de Discapacidad (REDIS) ${ }^{9}$ : Esta revista se crea como marco de divulgación de la investigación sobre discapacidad. Se trata de una revista electrónica de carácter científico, orientada a la publicación de artículos de investigación y/o de reflexión académica, científica y profesional en el ámbito de la discapacidad, desde una perspectiva multidisciplinar y en consonancia con el modelo social de la discapacidad y la visión de ésta como una cuestión de derechos humanos. Es una revista de acceso abierto, revisada por pares y dirigida a todas las personas y entidades que trabajan e investigan en este campo, que en sus primeros cinco años de vida ha publicado algunas de las principales investigaciones y artículos realizados en materia de discapacidad en nuestro país y en Latinoamérica.

- El Repositorio Iberoamericano sobre Discapacidad (RIBERDIS) ${ }^{10}$ : Al mismo tiempo que desarrollábamos REDIS, desde el CEDD pusimos en marcha Riberdis, que recopila y difunde la producción científica en formato digital y de acceso abierto que se produce en el ámbito iberoamericano en relación a los temas afines a la discapacidad. El principal objetivo de Riberdis es contribuir a la mejora de la difusión y del acceso al conocimiento mediante la organización, preservación y difusión de contenidos sobre discapacidad, agrupados en 12 comunidades temáticas.

\section{Conclusiones}

Con este texto he intentado describir la situación actual de la investigación social en materia de discapacidad y plantear, desde mi punto de vista, algunas propuestas y líneas estratégicas que seguir para impulsar esta investigación y articularla con las políticas públicas, la planificación y la intervención social.

En ese sentido, la investigación social ha de realizarse desde una visión de la discapacidad en línea con la Convención y la perspectiva de derechos y ha de servir para planificar las políticas públicas, diseñar actuaciones e implementarlas (así como posteriormente evaluarlas), dando a su vez respuesta a los preceptos marcados por la propia Convención.

9. Véase: https://www.cedd.net/redis.

10. Véase: http://riberdis.cedd.net/. 
Para ello, es importante establecer algunos criterios para decidir qué investigar, identificar temas relevantes y priorizarlos, aumentando y optimizando los recursos disponibles a esta labor.

En el plano técnico y metodológico, la investigación ha de ser comparativa y longitudinal, combinando las estrategias metodológicas cuantitativa y cualitativa, para abordar diferentes fenómenos sociales asociados a la discapacidad a lo largo del tiempo, desde la mayor complejidad y la más amplia perspectiva posible. Algunas tendencias y líneas estratégicas a priorizar podrían ser la creación de sistemas de indicadores sobre discapacidad e inclusión, la evaluación de políticas públicas y el desarrollo de estudios específicos de realidades emergentes.

Al mismo tiempo, hay que destacar que tan importante como producir conocimiento va a ser divulgarlo, difundirlo y generar transferencias con los ámbitos de la planificación y la intervención, pues la investigación social cobra sentido cuando transforma y mejora las políticas, las actuaciones profesionales y las prácticas sociales y, en definitiva, ayuda a lograr la inclusión social de las personas con discapacidad. 


\section{Referencias bibliográficas}

Agencia Tributaria (2016): Estadística sobre la discapacidad en las declaraciones del Impuesto sobre la Renta de las Personas Físicas (en línea). <https://www.agenciatributaria.es/AEAT.internet/datosabiertos/catalogo/hacienda/Estadistica_sobre_discapacidad_declaraciones_IRPF.shtml>, acceso 26 de septiembre de 2018.

Cea D’ Ancona, M. A. (1999): Metodología cuantitativa: estrategias y técnicas de investigación social. Madrid: Síntesis.

Comisión de Legislación del Real Patronato sobre Discapacidad (2012): Propuesta articulada de reforma del Código Civil y de la Ley de Enjuiciamiento Civil para su adecuación al artículo 12 de la Convención Internacional de los Derechos de las Personas con Discapacidad. Madrid: Fundación Aequitas y Real Patronato sobre Discapacidad.

Díaz Velázquez, E. (2017). El acceso a la condición de ciudadanía de las personas con discapacidad en España. Un estudio sobre la desigualdad por razón de discapacidad. Madrid: Ediciones Cinca.

Díaz Velázquez, E. (2010): "Ciudadanía, identidad y exclusión social de las personas con discapacidad”. Política y Sociedad, 47, (1): 115-135.

Dirección General de Políticas de Apoyo a la Discapacidad. (2014). Plan de Acción de la Estrategia Española de Discapacidad 2014-2020. Madrid: Real Patronato sobre Discapacidad.

Eurostat (2018): Eurostat Disability Database. (en línea). <https://ec.europa.eu/eurostat/web/health/disability/data/ database>, acceso 26 de septiembre de 2018.

Huete, A. (2013): "La exclusión de la población con discapacidad en España. Estudio específico a partir de la Encuesta Social Europea". Revista Española de Discapacidad, 1 (2): 7-24.

Jiménez, A. y Huete, A. (coords.) (2017): Informe Olivenza 2017, sobre la situación general de la discapacidad en España. Madrid: Observatorio Estatal de la Discapacidad.

Instituto Nacional de Estadística (2016): El Empleo de las personas con discapacidad (en línea). <http:// www.ine.es/dyngs/INEbase/es/operacion.htm?c=Estadistica_C\&cid=1254736055502\&menu=ultiDatos\&i $d p=1254735573175>$, acceso 26 septiembre de 2018 .

Instituto Nacional de Estadística (2016): El salario de las personas con discapacidad (en línea). <http:// www.ine.es/dyngs/INEbase/es/operacion.htm?c=Estadistica_C\&cid=1254736176911\&menu=ultiDatos\&i $d p=1254735573175>$, acceso 26 de septiembre de 2018 .

Instituto Nacional de Estadística(2012): Encuesta de Integración Socialy Salud (en línea). <https://www.ine.es/dyngs/ INEbase/es/operacion.htm?c=Estadistica_C\&cid=1254736176987\&menu=resultados\&idp=1254735573175>, acceso 26 de septiembre de 2018.

Ministerio deEducación (2018): Estadística deenseñanzas no universitarias. Necesidades de apoyo educativo. Curso 2016-17 (en línea), <http://estadisticas.mecd.gob.es/EducaDynPx/educabase/index.htm?type=pcaxis\&path=/ Educacion/Alumnado/Apoyo/Curso16-17/Acnee\&file=pcaxis\&l=s0>, acceso 26 de septiembre de 2018.

Organización de las Naciones Unidas (2008): Convención Internacional de los Derechos de las Personas con Discapacidad y protocolo facultativo. Resolución 61/106, aprobada por la Asamblea General el 13 de diciembre 
de 2006 (en línea) <http://www.un.org/disabilities/documents/convention/convoptprot-s.pdf>, acceso 26 de septiembre de 2018.

SIIS Centro de Documentación y Estudios (2014): La situación de las personas con discapacidad en el mercado laboral. Madrid: Observatorio sobre Discapacidad y Mercado de Trabajo en España, ODISMET. Fundación ONCE.

Washington Group on Disability Statistics: <http://www.washingtongroup-disability.com/>, acceso 26 de septiembre de 2018. 